\title{
Theory of Curie temperature enhancement in electron-doped EuO
}

\author{
Tobias Stollenwerk ${ }^{1,2}$ and Johann Kroha ${ }^{1,3, *}$ \\ ${ }^{1}$ Physikalisches Institut and Bethe Center for Theoretical Physics, \\ Universität Bonn, Nussallee 12, 53115 Bonn, Germany \\ ${ }^{2}$ German Aerospace Center, Linder Höhe, 51147 Cologne, Germany \\ ${ }^{3}$ Center for Correlated Matter, Zhejiang University, Hangzhou, Zhejiang 310058, China \\ (Received 10 September 2015; revised manuscript received 20 October 2015; published 17 November 2015)

\begin{abstract}
We present a comparative, theoretical study of the doping dependence of the critical temperature
\end{abstract} \\ $T_{C}$ of the ferromagnetic insulator-metal transitions in Gd-doped and O-deficient EuO, respectively. \\ The strong $T_{C}$ enhancement in $\mathrm{Eu}_{1-x} \mathrm{Gd}_{x} \mathrm{O}$ is due to Kondo-like spin fluctuations on the Gd sites, \\ which are absent in $\mathrm{EuO}_{1-x}$. Moreover, we find that the $T_{C}$ saturation in $\mathrm{Eu}_{1-x} \mathrm{Gd}_{x} \mathrm{O}$ for large \\ $x$ is due to a reduced activation of dopant electrons into the conduction band, in agreement with \\ experiments, rather than antiferromagnetic long-range contributions of the RKKY interaction. The \\ results shed light on possibilities for further increasing $T_{C}$. \\ PACS numbers: 71.30. $+\mathrm{h}, 75.20 . \mathrm{Hr}, 75.30 .-\mathrm{m}, 75.50 . \mathrm{Pp}$
}

\section{INTRODUCTION}

The demand for ever increasing speed and integrability of magnetic information storage devices as well as other spintronics applications calls for materials that are capable of transforming electronic or optical signals efficiently into magnetization and vice versa. Electrondoped europium monoxide $(\mathrm{EuO})$ is a promising candidate for this purpose, as it undergoes a simultaneous ferromagnetic (FM) and insulator-to-semimetal transition [1], exhibiting an outstanding magneto-electric response, including the strongest colossal magnetoresistance effect known [2,3], magneto-optical effects [4 7], and a spin polarization of the conduction band of nearly $100 \%$ in the FM state [8] 9]. Improved sample fabrication techniques 10,11 and europium monoxide's epitaxial integrability into Si [9] and GaAs [12] structures have renewed and intensified the interest in this material during the past few years.

Stoichiometric $\mathrm{EuO}$ is a wide band gap semiconductor with rocksalt structure. The local magnetic moments of $m=7 / 2 \mu_{B}$ situated in the Eu $4 f$ shell constitute a prototype Heisenberg ferromagnet, ordering ferromagnetically at the Curie temperature of $T_{C}=69 \mathrm{~K}$. Their interaction is mediated by virtual excitations (hybridization) of the tightly bound $\mathrm{Eu} 4 f$ electrons into the spatially more extended $\mathrm{Eu} 5 d$ orbitals and an exchange between the latter [13]. Upon electron doping the FM transition is accompanied by a simultaneous insulator-to-semimetal transition with a resistivity drop by 8 to 13 orders of magnitude [8, 14, 15]. Raising the transition temperature significantly is one of the major challenges involved in bringing the extraordinary properties of doped $\mathrm{EuO}$ to technological use.

Since early on, the general trend of $T_{C}$ being increased by electron-doping has been associated with the formation of magnetic polarons [16,17], i.e., conduction electrons dragging along a magnetic polarization cloud of local $\mathrm{Eu} 4 f$ moments. However, the experiments reveal more complex behavior. Gadolinium doping re- places Eu by $\mathrm{Gd}$ atoms, leaving the lattice of magnetic If moments intact and donating one additional electron per Gd atom from the Gd $5 d$ shell. In the doped material, $\mathrm{Eu}_{1-x} \mathrm{Gd}_{x} \mathrm{O}, T_{C}$ increases to values between $120 \mathrm{~K}$ and $170 \mathrm{~K}$ for $\mathrm{Gd}$ concentrations in the range of $x=0.04, \ldots, 0.08$ 5, 18 22], depending on the sample quality, strain, and measurement conditions 222. Invariably, $T_{C}$ saturates for higher $x$. Oxygen defects introduce two electrons per $\mathrm{O}$ defect, but only a weak $T_{C}$ increase has been observed in bulk, Eu-rich $\mathrm{EuO}_{1-x}$ [2, 3, 14, 15, 23, 24]. The $T_{C}$ increase reported in Ref. [25] for $\mathrm{EuO}_{1-x}$ may presumably be attributed [22] to the presence of a large external magnetic field inherently necessary for the SQUID measurement technique used.

The magnetic polaron theory alone cannot account for the $T_{C}$ saturation at high Gd concentration nor for the fact that $\mathrm{O}$ defects essentially do not raise $T_{C}$, even though they introduce twice as many carriers per impurity as Gd doping. It has been proposed [26,27] that the $T_{C}$ saturation might be understood in that, for increasing conduction-band filling, the oscillatory RKKY interaction [28 30] acquires increasingly antiferromagnetic (AF) contributions due to the decreasing RKKY wavelength. This requires, however, unrealistically high band filling. On the other hand, Hall resistivity measurements indicate [20] that the density of mobile charge carriers activated into the conduction band saturates in line with the $T_{C}$ saturation, providing a phenomenological reason for the limited $T_{C}$ increase in $\mathrm{Eu}_{1-x} \mathrm{Gd}_{x} \mathrm{O}$. In theoretical calculations, treating the $\mathrm{O}$ vacancies in a static approximation, Sinjukow and Nolting [31] found no increase of $T_{C}$ in $\mathrm{EuO}_{1-x}$ for an appropriate choice of system parameters. More sophisticated resummation techniques for the $\mathrm{Gd}$ impurity potential in $\mathrm{Eu}_{1-x} \mathrm{Gd}_{x} \mathrm{O}$ were able to correctly describe a shallow maximum of $T_{C}$ as a function of Gd concentration [32], but not the saturation of the mobile charge carrier density 20. Recent ab initio calculations [27,33] provided more quantitative results on the coupling parameters and spectral densities, but did not lead to a consistent understanding of all the experimental facts 
described above. Taking the local spin fluctuations on Gd impurities into account, Arnold and Kroha [34] could explain details of the magnetization behavior, the simultaneity of the insulator-semimetal transition and the $T_{C}$ increase in $\mathrm{Eu}_{1-x} \mathrm{Gd}_{x} \mathrm{O}$.

In this paper we report a comprehensive, theoretical study of the $T_{C}$ enhancement in electron-doped $\mathrm{EuO}$, extending the model of Ref. 34]. Gd impurities as well as $\mathrm{O}$ defects are treated dynamically as Anderson impurities hybridizing with the conduction band, however with strong or moderate on-site repulsion, respectively, ensuring the single or double occupancy of the Gd impurity or $\mathrm{O}$ vacancy orbitals. The direct comparison of the two cases shows that indeed the $T_{C}$ increase with Gd doping is caused by the Kondo-like spin fluctuations on the Gd sites and the concatenated accumulation of impurity spectral weight as well as conduction electron spectral weight at the chemical potential. This dynamical manybody effect drives the metallic transition, which, in turn, enhances the polaronic FM coupling between the Eu moments. By contrast, on $\mathrm{O}$ vacancies the two defect electrons form a spin-singlet, and local spin fluctuations are absent, leading only to a moderate $T_{C}$ enhancement due to a weak population of the conduction band. Moreover, the theory explains that in $\mathrm{Eu}_{1-x} \mathrm{Gd}_{x} \mathrm{O}$ the activation of charge carriers into the conduction band decreases with increasing doping concentration, in agreement with experiments [20], leading to the saturation of $T_{C}$. Including explicitly the RKKY interaction in our theory, we find that for all relevant temperatures $T$ and doping concentrations (band fillings) its long-distance AF contributions are negligible.

The paper is organized as follows. In Sec. II, we give a detailed justification of our model for $\mathrm{Eu}_{1-x} \mathrm{Gd}_{x} \mathrm{O}$ and $\mathrm{EuO}_{1-x}$ and describe the theory for its evaluation. The results are shown and discussed in Sec. III. We conclude in Sec. IV with a suggestion for a possible pathway to further enhance the transition temperature in electrondoped $\mathrm{EuO}$.

\section{THEORY}

\section{A. Model}

The model Hamiltonian for $\operatorname{Eu}_{1-x} \operatorname{Gd}_{x} \mathrm{O}$ as well as $\mathrm{EuO}_{1-x}$ reads,

$$
H=H_{0}+H_{c f}+H_{i m p} .
$$

The conduction band, comprised of the hybridizing $\mathrm{Eu}$ $5 d 6 s$ orbitals, is described by

$$
H_{0}=\sum_{\mathbf{k} \sigma}\left(\varepsilon_{\mathbf{k}}-\mu\right) c_{\mathbf{k} \sigma}^{\dagger} c_{\mathbf{k} \sigma},
$$

where $c_{\mathbf{k} \sigma}^{\dagger}$ is the conduction electron creation operator and $\varepsilon_{\mathbf{k}}$ the conduction band dispersion. $\mu$ is the chemical potential that fixes the total electron number (conduction and impurity electrons). In undoped $\mathrm{EuO}, \mu$ lies in the gap below the conduction band. The lattice of $\mathrm{Eu} 4 f$ moments is described by a Heisenberg Hamiltonian and a local coupling term between the Eu $4 f$ moments and the conduction electron spins,

$$
H_{c f}=-\sum_{\langle i, j\rangle} J_{i j} \mathbf{S}_{i} \cdot \mathbf{S}_{j}-J_{c f} \sum_{i} \boldsymbol{\sigma}_{i} \cdot \mathbf{S}_{i} .
$$

Here, $\mathbf{S}_{i}$ is the $4 f$ spin, $m_{S}=-7 / 2, \ldots,+7 / 2$, and $\boldsymbol{\sigma}_{i}=\frac{1}{2} \sum_{\sigma \sigma^{\prime}} c_{i \sigma}^{\dagger} \boldsymbol{\tau}_{\sigma \sigma^{\prime}} c_{i \sigma^{\prime}}$ is the conduction electron spin operator at site $i . J_{i j}>0$ is the direct exchange coupling between the localized moments which is independent of the conduction band occupation and therefore responsible for the Curie temperature of $69 \mathrm{~K}$ in stoichiometric $\mathrm{EuO} . J_{c f}$ is the exchange coupling between the $4 f$ moment and the conduction electron spin $\boldsymbol{\sigma}_{i}$ at lattice site $i$. The Gd impurities and $\mathrm{O}$ vacancies are described as Anderson impurities with a single electron binding energy $E_{d}<0$ and a hybridization with the conduction band, $V$

$$
\begin{aligned}
H_{c d} & =\left(E_{d}-\mu\right) \sum_{\{j\} \sigma} d_{j \sigma}^{\dagger} d_{j \sigma} \\
& +V \sum_{\{j\} \sigma}\left(c_{j \sigma}^{\dagger} d_{j \sigma}+d_{j \sigma}^{\dagger} c_{j \sigma}\right)+U \sum_{\{j\}} d_{j \uparrow}^{\dagger} d_{j \uparrow} d_{j \downarrow}^{\dagger} d_{j \downarrow},
\end{aligned}
$$

where $d_{j \sigma}^{\dagger}$ is the electron creation operator in an impurity or defect orbital at site $j$ and $\{j\}$ indicates a summation over the randomly placed impurity sites. In the following we will use the term impurity for both, Gd impurities and $\mathrm{O}$ vacancies, unless stated otherwise. The impurity number density will be denoted by $n_{I}$. Gd carries one extra electron in the $5 d$ shell as compared to Eu. Hence, $\mathrm{Gd}$ is in the strongly correlated regime with a strong onsite repulsion $U$ preventing double occupancy. Due to stoichiometry, an $\mathrm{O}$ vacancy attracts two extra electrons from the surrounding metal ions. Therefore, it is in the weakly correlated regime, with double occupancy in the ground state, i.e., a moderate onsite repulsion $0<U \ll$ $\left|E_{d}\right|$. The model, Eqs. (11)-(4), inherently incorporates the RKKY interaction [28 30] via 2nd-order, non-local perturbation theory in $J_{c f}$. Since, apart from RKKY effects, only local self-energies will be important for the following treatment of this paper, we here include the RKKY Hamiltonian explicitly,

$$
H^{R K K Y}=-\sum_{(i \neq j)}\left[K_{i j}^{\|} S_{i}^{z} S_{j}^{z}+K_{i j}^{\perp}\left(S_{i}^{x} S_{j}^{x}+S_{i}^{y} S_{j}^{y}\right)\right] .
$$

It is to be amended to the model Hamiltonian (1). A recollection of its derivation and the expressions for the coupling constants are given in Appendix A, see Eqs. (A8), (A9).

\section{B. Selfconsistent theory}

To evaluate this model, we follow and extend Ref. [34]. While the large spins of the $4 f$ Heisenberg lattice can 


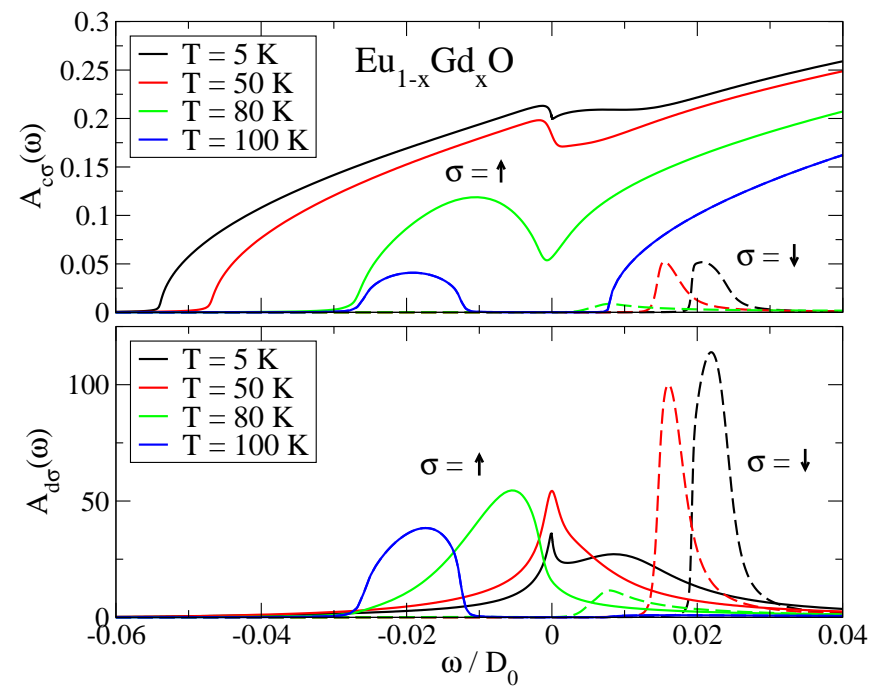

FIG. 1: (Color online) Conduction band (upper panel) and Gd impurity (lower panel) spectral density across the phase transition in $\mathrm{Eu}_{1-x} \mathrm{Gd}_{x} \mathrm{O}$ for $x=0.01 . T_{C} \approx 95 \mathrm{~K}$. Solid curves represent the majority $(\sigma=\uparrow)$ and dashed curves the minority $(\sigma=\downarrow)$ spin spectral density.

be treated in mean-field theory, it is essential to describe the Anderson impurities dynamically, in order to account for their spin and charge fluctuations. The conduction electron selfenergy induced by the impurities will be treated in a single-site approximation, i.e. it is given by the full impurity $T$ matrix times the impurity concentration $n_{I}$. This is valid for dilute impurities, where inter-impurity correlations are negligible. Writing the (retarded, $\omega \equiv \omega+i 0$ ) conduction electron Green's function as,

$$
G_{c \sigma}(\mathbf{k}, \omega)=\frac{1}{\omega+\mu-\varepsilon_{\mathbf{k}}-\Sigma_{c \sigma}(\omega)}
$$

yields for the total conduction selfenergy,

$$
\Sigma_{c \sigma}(\omega)=n_{I} V^{2} G_{d \sigma}(\omega)-\sigma J_{c f}\langle S\rangle, \quad \sigma= \pm \frac{1}{2},
$$

where $G_{d \sigma}(\omega)$ is the full Green's function of the impurity electrons. The expectation value of the $4 f$ spins is determined in mean-field theory by (with $\beta=1 / k_{B} T$, the inverse temperature),

$$
\langle S\rangle=\frac{\sum_{S=-\frac{7}{2}}^{\frac{7}{2}} S e^{\beta\left(2 J_{4 f}\langle S\rangle+J_{c f}\langle\sigma\rangle\right) S}}{\sum_{S=-\frac{7}{2}}^{\frac{7}{2}} e^{\beta\left(2 J_{4 f}\langle S\rangle+J_{c f}\langle\sigma\rangle\right) S}},
$$

Here, the conduction electron magnetization $\langle\sigma\rangle$ is calculated as,

$$
\langle\sigma\rangle=\frac{1}{2} \int d \omega f(\omega)\left[A_{c \uparrow}(\omega)-A_{c \downarrow}(\omega)\right],
$$

with $A_{c \sigma}(\omega)=-\frac{1}{\pi} \sum_{\mathbf{k}} \operatorname{Im} G_{c \sigma}(\mathbf{k}, \omega+i 0)$ the spindependent, interacting conduction electron density of

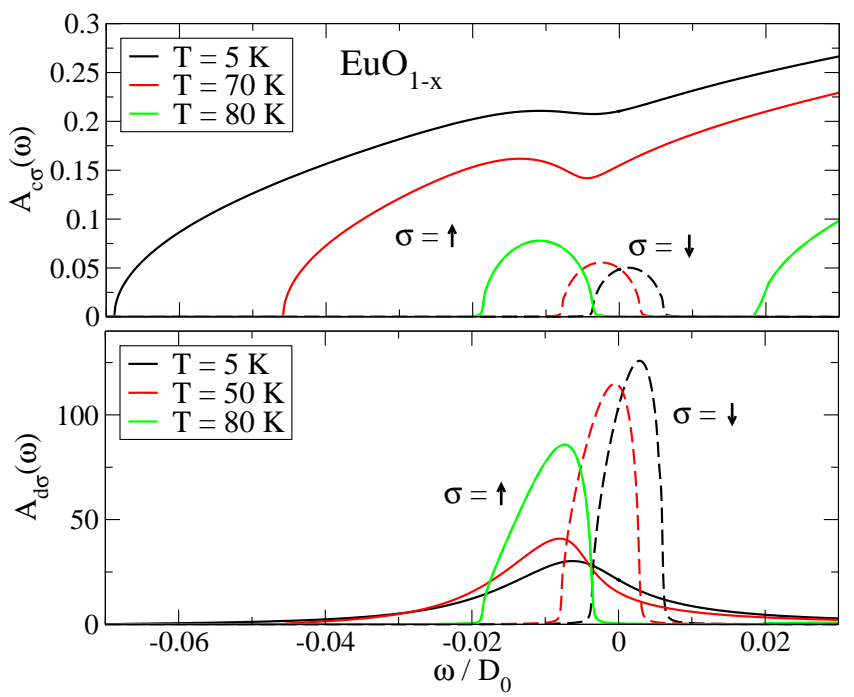

FIG. 2: (Color online) Conduction band (upper panel) and O vacancy (lower panel) spectral density across the phase transition in $\mathrm{EuO}_{1-x}$ for $x=0.01$. The bare O-defect parameters are $E_{d}=0.0 D_{0}, U=0$ (double occupancy). $T_{C} \approx 78 \mathrm{~K}$. Solid curves represent the majority $(\sigma=\uparrow)$ and dashed curves the minority $(\sigma=\downarrow)$ spin spectral density.

states (DOS). $J_{4 f}=\sum_{j} J_{0 j}$ is the effective mean-field coupling of the Heisenberg lattice. The short-range nature of $J_{i j}$ restricts the lattice summation essentially to the nearest neighbors of site $i=0$. The magnetic polaron effect 16 17] is incorporated in Eq. (8) via the conduction electron magnetization $\langle\sigma\rangle$. For later use, the conduction carrier density is given by, $n_{c}=\sum_{\sigma} \int d \omega f(\omega) A_{c \sigma}(\omega)$. The calculation of the local impurity Green's function $G_{d \sigma}(\omega)$ depends on whether the impurity is in the strongly (Gd impurities) or the weakly (O vacancies) correlated regime.

a. Gd impurities. We employ the auxiliary particle technique in non-crossing approximation [35 37] to describe the spin and charge fluctuations in the Gd $5 d$ orbitals. The limit $U \rightarrow \infty$ is taken, for simplicity, in order to prevent double occupancy. Since in $\mathrm{Eu}_{1-x} \mathrm{Gd}_{x} \mathrm{O}$ the DOS near the chemical potential is low or even vanishing, the Kondo temperature of the fluctuating spin, $T_{K}$, is far below $T_{C}$. The NCA is known to give reliable results for energies above and down to somewhat below $T_{K}$. In a magnetic field it produces, in addition to the Zeemansplit Kondo resonance, a spurious low-temperature singularity at the Fermi level for $T<T_{K}$. However, since $T_{K} \ll T_{C}$ in $\mathrm{Eu}_{1-x} \mathrm{Gd}_{x} \mathrm{O}$, the effect of this singularity is negligible for the temperature range relevant here. The NCA is also versatile enough to include an arbitrary energy dependence of the DOS. Therefore, it is the appropriate method for the present purpose [34]. The NCA equations, adapted for the $\mathrm{Eu}_{1-x} \operatorname{Gd}_{x} \mathrm{O}$ system with a gapped DOS and a non-trivial chemical potential, are given in Appendix B 
b. $O$ vacancies. The weak interaction on the $\mathrm{O}$ defects, where spin fluctuations are negligible, can be accounted for in the second-order perturbation theory in $U$. The (retarded) O-defect electron Green's function is $G_{d \sigma}(\omega)=1 /\left[\omega+\mu-E_{d}-\Sigma_{d \sigma}(\omega)\right]$, and the corresponding selfenergy reads, $\Sigma_{d \sigma}(\omega)=\Sigma_{d \sigma}^{(1)}(\omega)+\Sigma_{d \sigma}^{(2)}(\omega)$, where

$$
\begin{aligned}
\Sigma_{d \sigma}^{(1)}(\omega)= & \pi V^{2} A_{c \sigma}(\omega)+U \int d \varepsilon f(\varepsilon) A_{d-\sigma}(\varepsilon) \\
\operatorname{Im} \Sigma_{d \sigma}^{(2)}(\omega)= & \\
-U^{2} \int d \varepsilon_{1} \int & d \varepsilon_{2} A_{d \sigma}\left(\varepsilon_{1}+\omega\right) A_{d-\sigma}\left(\varepsilon_{1}+\varepsilon_{2}\right) A_{d-\sigma}\left(\varepsilon_{2}\right) \\
& \times\left[b\left(\varepsilon_{1}\right)+f\left(\varepsilon_{1}+\omega\right)\right]\left[f\left(\varepsilon_{2}\right)-f\left(\varepsilon_{1}+\varepsilon_{2}\right)\right]
\end{aligned}
$$

where $f(\omega)$ and $b(\omega)$ are Fermi and Bose distribution functions, respectively, and $\operatorname{Re} \Sigma_{d \sigma}^{(2)}(\omega)$ is given by the Kramers-Kronig relation.

Long-range RKKY coupling. When the RKKY interaction is included, the $4 f$ Heisenberg coupling is changed to $J_{i j} \rightarrow J_{i j}+K_{i j}$. In the above equations this leads to a modified mean-field coupling,

$$
J_{4 f}=\sum_{j n . n .0} J_{0 j}+\sum_{j \neq 0} K_{0 j}^{\|} .
$$

The lattice summation in the second term is carried out over the fcc lattice of the $\mathrm{EuO}$ rocksalt structure. Note that on mean-field level only the longitudinal RKKY component contributes and can give FM as well as AF contributions to the total coupling. $K_{0 j}^{\|}$involves nonlocal Green's functions (c.f. Appendix A and, hence, the band dispersion $\varepsilon_{\mathbf{k}}$. For simplicity and since anisotropy effects are not important in bulk EuO, we assume for the RKKY interaction an isotropic dispersion which is constructed such [38] that it reproduces the bare conduction DOS.

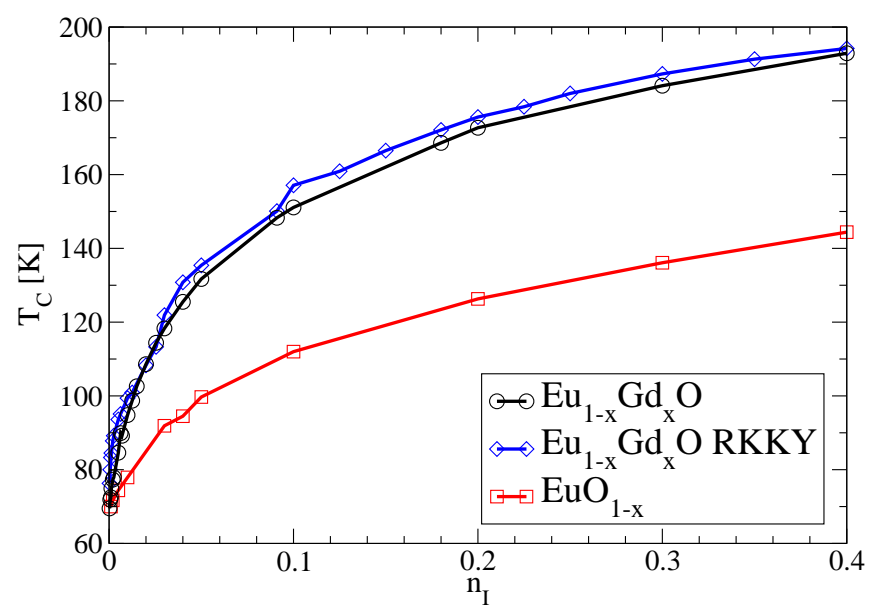

FIG. 3: (Color online) Curie temperature vs. the doping concentration $n_{I}$ for Gd-doped and O-deficient EuO. Bare parameters for $\mathrm{O}$ vacancies: $E_{d}=0.0 D_{0}, U=0$. The blue curve represents the behavior for $\mathrm{Eu}_{1-x} \mathrm{Gd}_{x} \mathrm{O}$ including the RKKY interaction, see text.

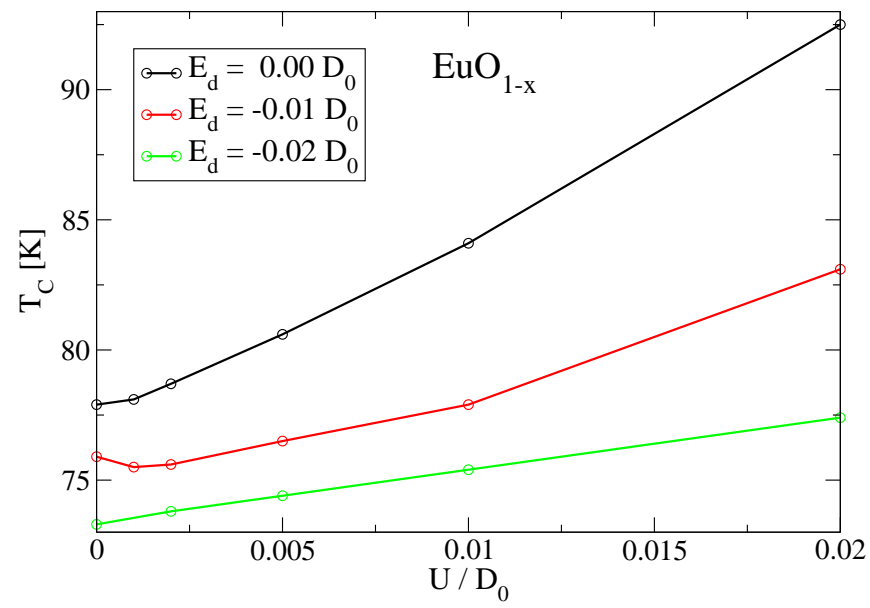

FIG. 4: (Color online) Curie temperature vs. on-site Coulomb repulsion $U$ in $\mathrm{EuO}_{0.99}$ for various impurity level energies $E_{d}$. It is seen that for more tightly bound defect electrons the $T_{C}$ enhancement in $\mathrm{EuO}_{a-x}$ is even weaker than for the parameter values of Fig. 3 .

The system is subject to the doping condition that the total density of charge carriers is $n_{t o t}=n_{I}$ for $\mathrm{Eu}_{1-x} \mathrm{Gd}_{x} \mathrm{O}$ and $n_{t o t}=2 n_{I}$ for $\mathrm{EuO}_{x}$. That is,

$$
\sum_{\sigma} \int d \omega f(\omega)\left[A_{c \sigma}(\omega)+n_{I} A_{d \sigma}(\omega)\right]-n_{t o t}=0
$$

The selfconsistent set of equations (6) -13), in the case of $\mathrm{Eu}_{1-x} \mathrm{Gd}_{x} \mathrm{O}$ amended by the NCA equations (B1)(B3), is solved by iteration, where in each iteration step the chemical potential $\mu$ is adjusted so as to fulfill the particle number constraint (13). Note that the RKKY coupling strength $K_{i j}^{\|}$, Eq. (A9), is also determined selfconsistently via the interacting conduction electron propagators.

\section{RESULTS}

\section{A. Parameter values}

For the numerical calculations below, we choose a semi-elliptic DOS for the non-interacting conduction band of stoichiometric EuO, $N_{c \sigma}^{(0)}(\omega)=$ $\left(2 / \pi D_{0}\right) \sqrt{\left(\omega-\mu_{0}-D_{0}\right)^{2} / D_{0}^{2}-1}$. The conduction half-bandwidth is taken to be $D_{0}=8 \mathrm{eV}$, and the chemical potential of the undoped system lies in the gap below the conduction band, $\mu_{0}=-0.02 D_{0}$, consistent with experiments [8]. All energies are measured relative to the (interacting) chemical potential $\mu$ and are given in units of $D_{0}$. The mean-field Heisenberg coupling $J_{4 f}$ (without RKKY interaction) is chosen such that $T_{C}=69 \mathrm{~K}$ is obtained for undoped EuO. This yields $J_{4 f}=7 \cdot 10^{-5} D_{0}$ [34]. $J_{c f}$ is much larger than $J_{4 f}$, because the overlap between the neighboring $\mathrm{Eu} 4 f$ orbitals is much smaller 


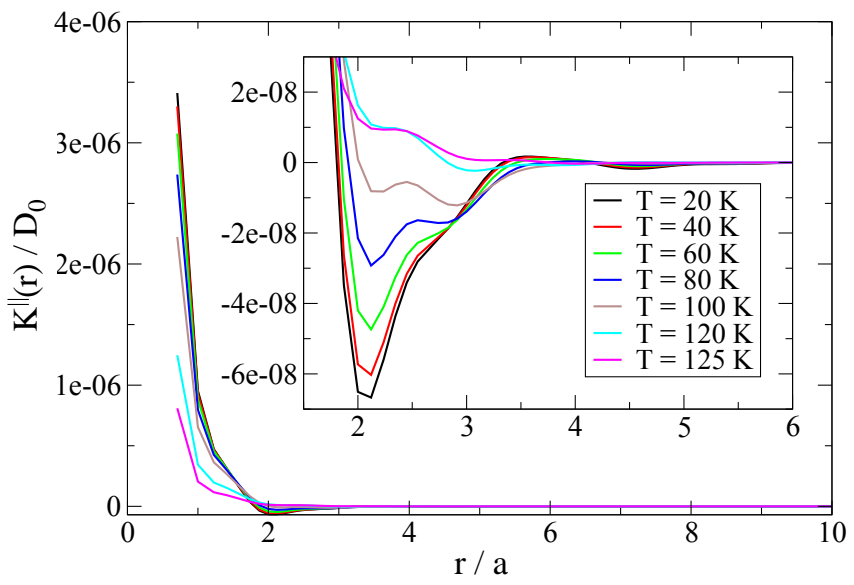

FIG. 5: (Color online) Longitudinal RKKY coupling $K^{\|}(r)$ for $x=n_{I}=0.04$ and various temperatures in $\mathrm{Eu}_{1-x} \mathrm{Gd}_{x} \mathrm{O}$. The inset expands the AF region around two to four lattice spacings a of the FCC lattice.

than their overlap with the conduction wave functions. From the spatial separation of the Eu $4 f$ orbitals the ratio $J_{c f} / J_{4 f}$ is roughly estimated to give $J_{c f}=0.05 D 0$, see also Ref. [34]. This also determines the RKKY coupling strength via Eq. (A9). We fix the bare parameters of a Gd impurity such that for $T=0$ and vanishing impurity concentration its occupation is $n_{d} \approx 1$ and that the impurity electron gets thermally activated in the experimentally relevant temperature range. This yields, $E_{d}=0.0$, $\Gamma:=\pi V^{2} / D_{0}=0.05 D_{0}$, and $U \rightarrow \infty$. Note that hybridization and interaction substantially renormalize the impurity level, $E_{d} \rightarrow \tilde{E}_{d} \approx-0.02 D_{0}$ (Haldane shift 39], see also Fig. 1), so that $n_{d} \approx 1$ is realized in the $n_{I} \rightarrow 0$ limit. This also renders the $T_{K}$ of the impurity far below $T_{C}$, since in our system the DOS at the Fermi level $E_{F}$ remains always $A_{c \sigma}(0) \ll 1 / D_{0}$. For $\mathrm{O}$ vacancies, in absence of more detailed information about their structure other than double occupancy, we set the effective hybridization $\Gamma=0.05 D_{0}$, as for $\mathrm{Gd}$, and perform a scan of $E_{d} \leq 0$ and $U$ within the bound-state, weakly correlated regime, see below. The RKKY interaction will be included and discussed in Subsec. [IIC only.

\section{B. Gadolinium impurities vs. Oxygen vacancies}

Figures 1 and 2 show the evolution of the conduction band and impurity spectral densities across the phase transition for low-doped $\mathrm{Eu}_{1-x} \mathrm{Gd}_{x} \mathrm{O}$ and $\mathrm{EuO}_{1-x}$, respectively. For both, $\mathrm{Eu}_{1-x} \mathrm{Gd}_{x} \mathrm{O}$ and $\mathrm{EuO}_{1-x}$, in the high-temperature insulating phase the spin degenerate conduction DOS is comprised of a large, unoccupied band and a small side band which is induced by the hybridization with the impurity orbitals and is centered around the impurity binding energy $E_{d}$, lying entirely below $\mu$ and, therefore, not contributing to the conductivity. As the temperature is lowered, in $\mathrm{Eu}_{1-x} \mathrm{Gd}_{x} \mathrm{O}$ the impu-

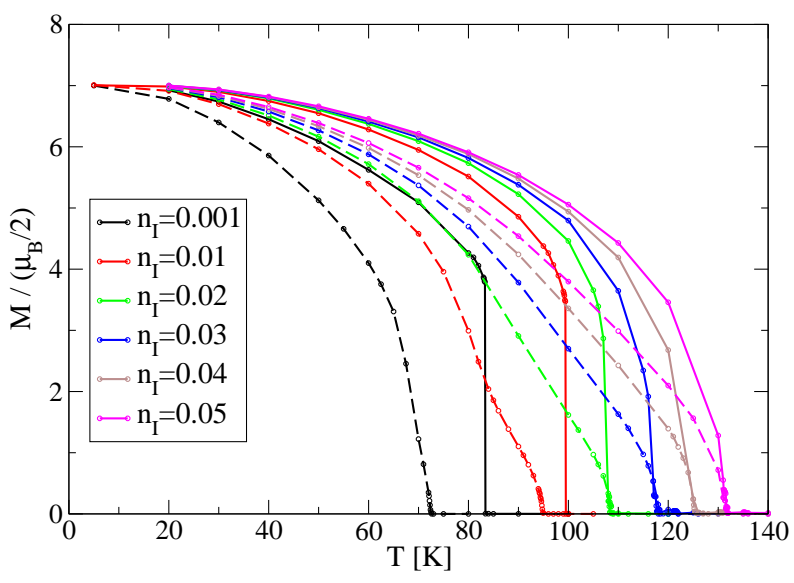

FIG. 6: (Color online) Total magnetization $M=\langle S\rangle+$ $\langle\sigma\rangle$ vs. temperature $T$ for various doping concentrations in $\mathrm{Eu}_{1-x} \mathrm{Gd}_{x} \mathrm{O}$ with long range RKKY coupling (solid curves) and without RKKY coupling (dashed curves).

rity spectrum accumulates spectral weight at the chemical potential which eventually develops into a peak at $\omega=0$ (Fig. 1). Below $T_{C}$ the spectral densities are split into majority and minority bands. Our NCA calculations show that this is due to local, Kondo-like spin fluctuations in the $\mathrm{Gd} 5 d$ orbitals [34]. Because of hybridization, the conduction electron DOS develops spectral weight at $\omega=0$ as well, and the side band merges with the main conduction band. This drives the metallic transition and simultaneously enhances, via the magnetic polaron effect [c.f. Eq. (8)], the FM transition temperature as well. In $\mathrm{EuO}_{1-x}$, the local spin fluctuation effect is absent. Here, the metallic transition occurs only when the conduction side band is eventually broadened and shifted, via hybridization with the $\mathrm{O}$ vacancy band enough to gain overlap with the chemical potential (Fig. 2), leading to a much lower $T_{C}$ than in $\operatorname{Eu}_{1-x} \operatorname{Gd}_{x} \mathrm{O}$. In Fig. 3 the doping-dependent $T_{C}$ enhancement is compared for magnetic $\mathrm{Gd}$ impurities and non-magnetic $\mathrm{O}$ vacancies (black and red curves). Here, for $\mathrm{O}$ vacancies, $U=0$ (double occupancy) and otherwise the same parameter values as for $\mathrm{Eu}_{1-x} \mathrm{Gd}_{x} \mathrm{O}$ are assumed. This allows for a direct assessment of the importance of low-lying, local spin fluctuations for the $T_{C}$ enhancement. The essential role of on-site correlations as well as conduction electron doping is further substantiated by Fig. 4. where $T_{C}$ is shown for varying $U$ and $E_{d}$ values in $\mathrm{EuO}_{1-x}: T_{C}$ is enhanced by repulsive onsite correlations (increasing $U)$ and is reduced by the dopant electrons more tightly bound to the defect (more negative $E_{d}$ ).

\section{RKKY interaction in $\mathbf{E u}_{1-x} \mathbf{G d}_{x} \mathbf{O}$}

We now study the influence of the long-range RKKY interaction on the phase transition in $\mathrm{Eu}_{1-x} \mathrm{Gd}_{x} \mathrm{O}$. For short Fermi wavelength, the RKKY interaction might 
make an AF contribution to the total coupling and, thus, lead to the experimentally observed saturation of $T_{C}$ [5, 18 22], as has been suggested in Ref. [26]. In order to analyze the possible influence of the RKKY interaction on the saturation at high doping concentration, we now adjust the value of $J_{c f}$ such that the theory including RKKY reproduces the previous results without RKKY interaction (Sec. IIIB) in the low-doping regime, and will compare the results at high doping. This yields the new value $J_{c f}=0.0405 D_{0}$. The RKKY coupling $K^{\| l}(r)$, selfconsistently calculated for the interacting system, is displayed in Fig. [5 as a function of distance $r$ for a typical Gd doping concentration of $n_{I}=0.04$ over the complete, relevant $T$ range. It shows weak $\mathrm{AF}$ behavior only in the range of about 2 to 4 FCC lattice constants. The resulting total magnetization $M$ is shown in Fig. 6. While the FM magnetization is even enhanced by $K^{\|}$below the transition, it does not substantially alter $T_{C}$, especially for higher doping. This is plausible, because the RKKY interaction is not active for $T>T_{C}$ (empty conduction band), but its long-range, overall FM behavior enhances $M$ once the band is populated for $T<T_{C}$. Such enhancement of the FM coupling by an RKKY-like interaction is consistent with recent experiments on $\mathrm{EuO}$ doped with non-magnetic La atoms [40]. Fig. 3 directly compares $T_{C}$ with and without RKKY coupling (blue and black curves, respectively) in our calculation. It is seen that including the RKKY interaction and reducing the direct exchange coupling to $J_{c f}=0.0405 D_{0}$ not only reproduces the $T_{C}$ behavior at small $n_{I}$ (by construction), but also does not change the behavior for the largest $n_{I}$ considered. For the small band fillings relevant in $\mathrm{Eu}_{1-x} \mathrm{Gd}_{x} \mathrm{O}$ the effects of the RKKY interaction are essentially doping independent and can be absorbed in a proper choice of $J_{c f}$, at least as far as $T_{C}$ in bulk systems is concerned. We conclude that the experimentally observed $T_{C}$ saturation behavior in $\mathrm{Eu}_{1-x} \mathrm{Gd}_{x} \mathrm{O}$ for large $n_{I}$ cannot be attributed to the RKKY interaction. Note that $a b$ initio calculations 27, 33. presumably overestimate the antiferromagnetic contributions from the RKKY interaction, because they do not take the Kondo-like spin fluctuations on the Gd sites and the resulting accumulation of spectral weight at the chemical potential into account. As a consequence, the RKKY wavelength comes out too short and, hence, its antiferromagnetic contributions too large. This may be the origin why these calculations overestimate the decrease of $T_{C}$ for large doping concentration as compared to experiments [20,21].

\section{Dopant activation and $T_{C}$ saturation}

In Fig. 7 we show the charge carrier concentration in the conduction band $n_{c}$ (number of carriers per lattice unit cell; inset) as well as the dopant activation $n_{c} / n_{I}$ as a function of impurity concentration $n_{I}$ at the lowest temperature considered, $T=5 \mathrm{~K}$, similar to Ref. [20]. For low doping the impurity spectral weight $A_{d \sigma}(\omega)$ (both,

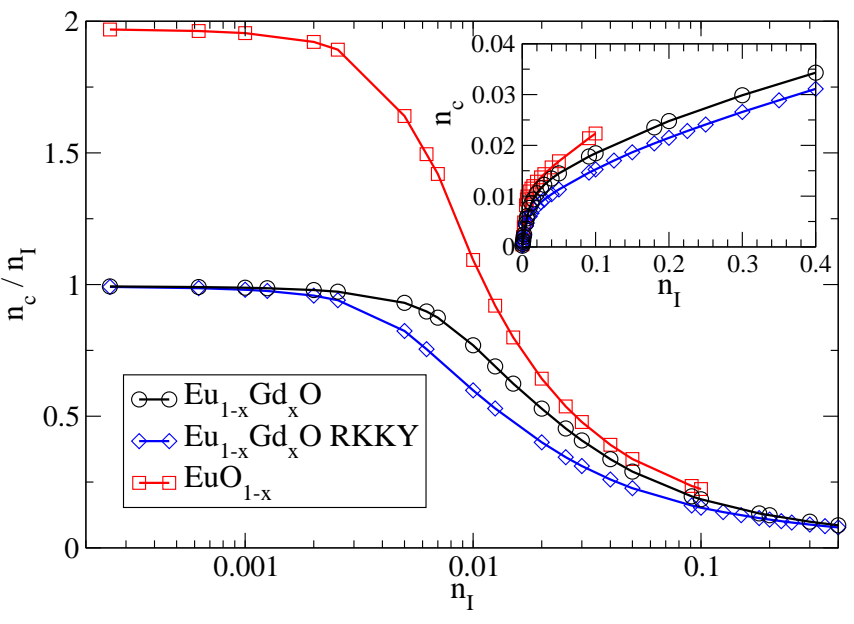

FIG. 7: (Color online) Dopant activation $n_{c} / n_{I}$ for Gd doped and $\mathrm{O}$ deficient $\mathrm{EuO}$ at the lowest temperature considered, $T=5 K$. The carrier concentration $n_{c}$ is shown in the inset.

$\sigma=\uparrow, \downarrow)$ is almost entirely shifted above $E_{F}$ at this temperature, as can bee seen from Figs. 1, 2, lower panels, so that the impurity level is completely emptied into the conduction band. Consequently, the dopant activation is $n_{c} / n_{I}=1(\mathrm{Gd})$ or $n_{c} / n_{I}=2$ (O vacancies) up to a doping concentration of about $n_{I}=0.01$ (Fig. 7). For higher $n_{I}$, the impurity contribution to the conduction electron selfenergy, Eq. (7), gets increasingly enhanced by the disorder. Via the Kramers-Kronig relation for the real part of $\Sigma_{c \sigma}(\omega)$ this implies a downward shift of the conduction side band and, connected with it, a downward shift of the impurity band below $E_{F}$. This is seen in Fig. 8 , It results in a re-population of the impurity levels and a crossover to a reduced $n_{c} / n_{I}$, as seen in Fig. (7) Note that the description of the Gd impurities as Anderson impurities with spin fluctuations is crucial for the downward shift of the impurity levels. The reduction of the dopant activation $n_{c} / n_{I}$ is in agreement with the experimental findings of Ref. [20]. Note that in Ref. [20] a reduced dopant activation is also found in the limit of small $n_{I}$. Presumably this is, because their Hall measurements determine the mobile carrier density $n$ only. However, for small impurity concentration, part of the electrons in the conduction band will be bound around the impurity locations. However, all electrons in the conduction band, given by $n_{c}$, contribute to the electron-enhanced magnetic coupling. Comparing the doping dependence of $n_{c}$ in the inset of Fig. 7 with $T_{C}$ in Fig. 3 shows that the latter follows the behavior of $n_{c}$. Displaying now $T_{C}$ (same data as in Fig. 3) as a function of the carrier concentration $n_{c}$ in Fig. 9 shows that it grows essentially linearly with $n_{c}$, showing only a slightly decreasing slope for the highest $n_{c}$. Note that the highest carrier concentration of $n_{c} \approx 0.04$ appears experimentally achievable, while the corresponding doping concentration of $n_{I}=0.4$ is not, due to stability reasons of the crystal structure. The saturation-like behavior of $T_{C}$ for large $n_{I}$ doping 


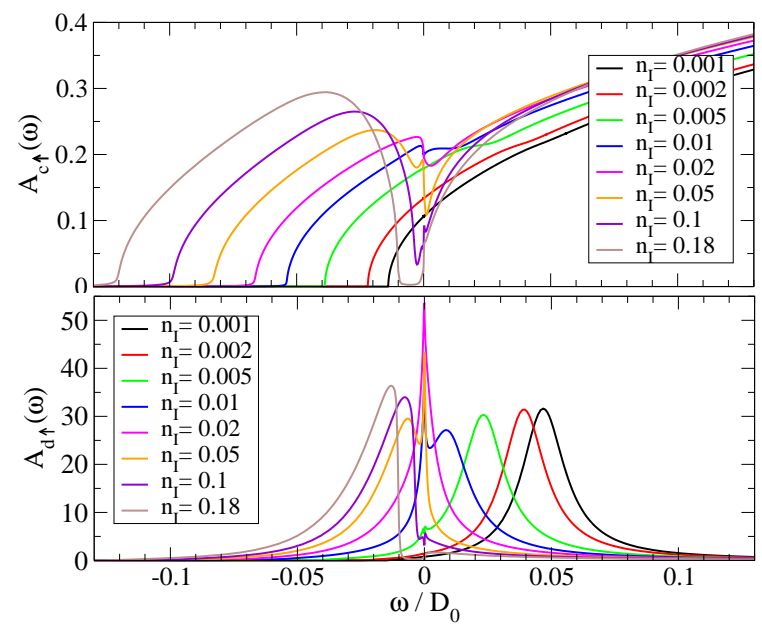

FIG. 8: (Color online) Majority spectral densities for conduction electrons (upper panel) and impurity electrons (lower panel) in $\mathrm{Eu}_{1-x} \mathrm{Gd}_{x} \mathrm{O}$ at $T=5 \mathrm{~K}$ for various impurity concentrations $n_{I}$. The figure shows the downward shift of the impurity levels with increasing $n_{I}$.

is, therefore, to be considered a consequence of the reduced dopant activation for large doping, in complete agreement with the conclusion of Ref. [20].

\section{CONCLUSION}

We have performed a detailed comparison of the FM insulator-metal transitions in $\mathrm{Eu}_{1-x} \mathrm{Gd}_{x} \mathrm{O}$ and in $\mathrm{EuO}_{1-x}$, respectively, using a model that treats the dopant impurities as Anderson impurities in the strongly (Gd) or weakly (O vacancies) correlated regime, and that had previously provided a detailed description [34] of experimental magnetization, resistivity and total conduction band polarization data. Our results show that for a significant, doping-induced $T_{C}$ enhancement a cooperation of two effects is necessary, (1) Kondo-like, low-energy spin fluctuations accumulating impurity as well as conduction spectral weight at the Fermi energy and (2) efficient population of this low-lying spectral weight and subsequent enhancement of the FM interaction between the $4 f$ moments mediated by the conduction electrons. In addition, our calculations provide evidence that the tendency of $T_{C}$ to saturate for high doping concentrations is not due to AF contributions of the RKKY interaction but rather due to a limitation of the dopant electron activation into the conduction band, confirming experimental results [20]. Hence, an increase of $T_{C}$ beyond the presently achievable values may be possible, if only the conduction band can be populated in a more efficient way. This is in line with recent pump-probe experiments [7] where enhanced FM coupling was achieved by photodoping into the conduction band [41]. The combination of all these findings point to a possible pathway to further enhancement of $T_{C}$ : the magnetic impurities

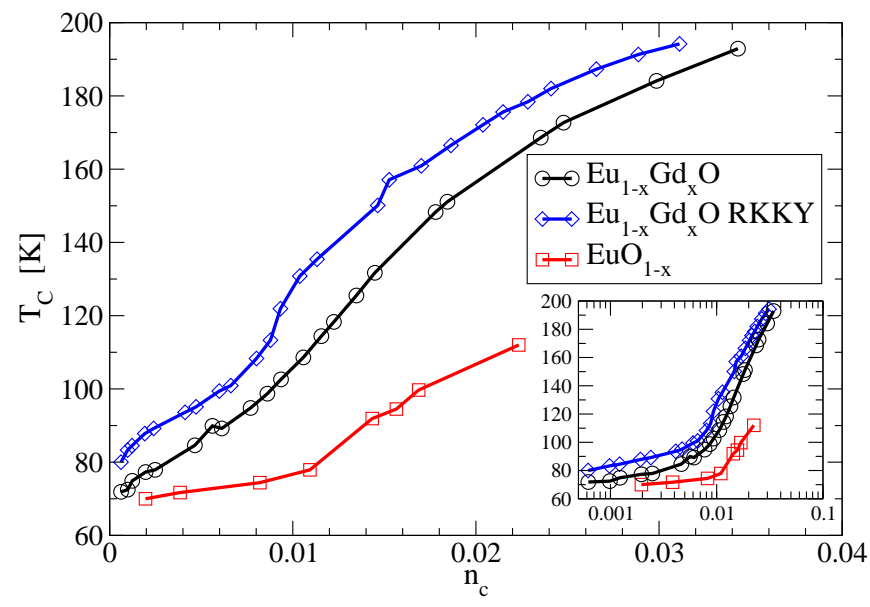

FIG. 9: (Color online) Curie temperature vs. carrier concentration $n_{c}$ in the conduction band for Gd-doped and Odeficient EuO. (Inset) Semilogarithmic plot of the data.

generating low-energy spin fluctuations and the chargedoping impurities need not necessarily be of the same type. More efficient carrier doping may be achievable by using different types of donor atoms (with impurity levels closer or above the Fermi energy) in addition to Gd, or by carrier coupling at interfaces in heterostructures.

\section{ACKNOWLEDGMENTS}

We gratefully acknowledge useful discussions with Carsten Busse, Manfred Fiebig, Jürgen Klinkhammer, Jochen Mannhart, Andreas Schmehl and Hao Tjeng. This work was supported in part by the Deutsche Forschungsgemeinschaft through SFB 608.

\section{Appendix A: RKKY interaction in paramagnetic metals and semimetals}

In this appendix we derive the expressions for the RKKY interaction, allowing for an arbitrary magnetization of the conduction band and then specializing for the paramagnetic case (vanishing magnetization) and the semimetallic case (complete magnetization). The interaction Hamiltonian between localized spins $\mathbf{S}_{i}$ at sites $i$ and the conduction electron spins reads,

$$
H_{c f}=-J_{c f} \sum_{i} \mathbf{S}_{i} \cdot \mathbf{s}_{i}
$$

where $\mathbf{s}_{i}=1 / 2 \sum_{\sigma \sigma^{\prime}} c_{i \sigma}^{\dagger} \boldsymbol{\sigma} c_{i \sigma^{\prime}}$ is the conduction electron spin operator at site $i$ and $\boldsymbol{\sigma}=\left(\sigma^{x}, \sigma^{y}, \sigma^{z}\right)$ the vector of Pauli matrices. Evaluating the time evolution of the conduction electrons in the presence of another localized spin $\mathbf{S}_{j}$ according to $\exp \left[-\int_{0}^{\beta} d \tau H_{c f}(\tau)\right]$ in first order of the spin coupling $J_{c f}$ and tracing out the conduction electron degrees of freedom, one obtains in the static limit 
( $\omega=0)$ the RKKY interaction operator between the local spins $\mathbf{S}_{i}$ and $\mathbf{S}_{j}$,

$$
H_{i j}^{R K K Y}=-\left.J_{c f}^{2}\left\langle\left(\mathbf{S}_{i} \cdot \mathbf{s}_{i}\right)\left(\mathbf{S}_{j} \cdot \mathbf{s}_{j}\right)\right\rangle_{c}\right|_{\omega=0} .
$$

Here $\langle(\ldots)\rangle_{c}:=\operatorname{tr}_{c}\left\{\mathrm{e}^{-\beta H}(\ldots)\right\} / Z_{G}$ denotes the thermal trace over the conduction electron Hilbert space. Using Wick's theorem, it can be written as,

$$
H_{i j}^{R K K Y}=-\frac{J_{c f}^{2}}{4} \sum_{\alpha, \beta=x, y, z} \sum_{\sigma \sigma^{\prime}} S_{i}^{\alpha} \sigma_{\sigma \sigma^{\prime}}^{\alpha} \sigma_{\sigma^{\prime} \sigma}^{\beta} S_{j}^{\beta} \Pi_{i j}^{\sigma \sigma^{\prime}}(0),
$$

where $\Pi_{i j}^{\sigma \sigma^{\prime}}$ is the conduction electron density propagator between the sites $i$ and $j$ as depicted diagrammatically in Fig. 10, It has the general form,

$$
\Pi_{i j}^{\sigma \sigma^{\prime}}(i \omega)=-\frac{1}{\beta} \sum_{\varepsilon_{n}} G_{j i \sigma}\left(i \varepsilon_{n}+i \omega\right) G_{i j \sigma^{\prime}}\left(i \varepsilon_{n}\right) .
$$

In the static limit it reads,

$$
\begin{aligned}
\Pi_{i j}^{\sigma \sigma^{\prime}}(0)= & -\int d \varepsilon f(\varepsilon) \times \\
& {\left[A_{i j \sigma}(\varepsilon) \operatorname{Re} G_{i j \sigma^{\prime}}(\varepsilon)+A_{i j \sigma^{\prime}}(\varepsilon) \operatorname{Re} G_{i j \sigma}(\varepsilon)\right], }
\end{aligned}
$$

where $A_{i j \sigma}(\varepsilon)=-\operatorname{Im} G_{i j \sigma}(\varepsilon+i 0) / \pi$. Performing the spin contractions in Eq. (A3) and defining the longitudinal and the transverse polarization functions, respectively, as

$$
\begin{aligned}
\Pi_{i j}^{\| !}(0) & =\frac{1}{2} \sum_{\sigma} \Pi_{i j}^{\sigma \sigma}(0) \\
& =-\sum_{\sigma} \int d \varepsilon f(\varepsilon) A_{i j \sigma}(\varepsilon) \operatorname{Re} G_{i j \sigma}(\varepsilon) \\
\Pi_{i j}^{\perp}(0) & =\frac{1}{2} \sum_{\sigma} \Pi_{i j}^{\sigma-\sigma}(0) \\
& =-\sum_{\sigma} \int d \varepsilon f(\varepsilon) A_{i j \sigma}(\varepsilon) \operatorname{Re} G_{i j-\sigma}(\varepsilon),
\end{aligned}
$$

one obtains the RKKY interaction Hamiltonian,

$$
\begin{aligned}
H^{R K K Y} & =\sum_{(i, j)} H_{i j}^{R K K Y} \\
& =-\sum_{(i, j)}\left[K_{i j}^{\|} S_{i}^{z} S_{j}^{z}-K_{i j}^{\perp}\left(S_{i}^{x} S_{j}^{x}+S_{i}^{y} S_{j}^{y}\right)\right]
\end{aligned}
$$

where the sum runs over all (arbitrarily distant) pairs of localized spins $\mathbf{S}_{i}$ and $\mathbf{S}_{j}$, and

$$
K_{i j}^{\|}=\frac{1}{2} J_{c f}^{2} \Pi_{i j}^{\|}(0), \quad K_{i j}^{\perp}=\frac{1}{2} J_{c f}^{2} \Pi_{i j}^{\perp}(0),
$$

are the longitudinal and transverse RKKY couplings, respectively. As seen from Eqs. (A8) and (A9), the RKKY



FIG. 10: Diagram for the spin-dependent conduction electron polarization function $\Pi_{i j}^{\sigma \sigma^{\prime}}(\omega)$, generating the RKKY interaction. The solid lines represent conduction electron propagators.

interaction is in general anisotropic for a magnetized conduction band.

We now present explicitly the expressions for the special cases of a paramagnet and of a semimetal. For a paramagnetic conduction band we have $G_{i j \sigma}=G_{i j,-\sigma}$ independent of spin. Hence, the RKKY coupling is isotropic, and we have the paramagnetic RKKY Hamiltonian,

$$
H_{P M}^{R K K Y}=-\sum_{(i, j)} K_{i j}^{P M} \mathbf{S}_{i} \cdot \mathbf{S}_{j}
$$

with

$$
K_{i j}^{P M}=-\frac{J_{c f}^{2}}{2} \sum_{\sigma} \int d \varepsilon f(\varepsilon) A_{i j \sigma}(\varepsilon) \operatorname{Re} G_{i j \sigma}(\varepsilon) .
$$

For a semimetal, i.e., for a completely spin-magnetized conduction band with majority spin $\sigma=\uparrow$ we have $A_{i j \downarrow}(\varepsilon)=0$, and the semimetallic RKKY Hamiltonian reads,

$$
\begin{aligned}
H_{F M}^{R K K Y}=-\sum_{(i, j)} & {\left[K_{i j}^{F M \|} S_{i}^{z} S_{j}^{z}\right.} \\
+ & \left.K_{i j}^{F M \perp}\left(S_{i}^{x} S_{j}^{x}+S_{i}^{y} S_{j}^{y}\right)\right],
\end{aligned}
$$

with

$$
\begin{aligned}
K_{i j}^{F M \|} & =-\frac{J_{c f}^{2}}{2} \int d \varepsilon f(\varepsilon) A_{i j \uparrow}(\varepsilon) \operatorname{Re} G_{i j \uparrow}(\varepsilon) \\
K_{i j}^{F M \perp} & =-\frac{J_{c f}^{2}}{2} \int d \varepsilon f(\varepsilon) A_{i j \uparrow}(\varepsilon) \operatorname{Re} G_{i j \downarrow}(\varepsilon) .
\end{aligned}
$$

The missing spin summation in Eqs. A13, A14 as compared to Eq. A11 indicates that in the completely magnetized band only the majority spin species contributes to the coupling. Note, however, that the transverse coupling $J_{F M \perp}^{R K K Y}$ ij still non-zero even in the ferromagnetically saturated case because of virtual (off-shell) minority spin contributions represented by the real part, $\operatorname{Re} G_{i j \downarrow}(\varepsilon)$ in Eq. (A14). The Curie temperature $T_{C}$, where the band magnetization vanishes, is controlled by the paramagnetic RKKY coupling, Eq. (A10), while far below $T_{C}$ the carrier concentration $n_{C}$ is determined by the semimagnetic RKKY coupling, Eq. A12). 


\section{Appendix B: NCA equations}

The selfconsistent NCA equations for the pseudofermion $(f)$ and slave boson $(b)$ self-energies read $(\omega \equiv \omega+i 0)$

$$
\begin{aligned}
\Sigma_{f \sigma}(\omega) & =V^{2} \int d \varepsilon[1-f(\varepsilon)] A_{c \sigma}(\varepsilon) G_{b}(\omega-\varepsilon) \\
\Sigma_{b}(\omega) & =V^{2} \sum_{\sigma} \int d \varepsilon f(\varepsilon) A_{c \sigma}(\varepsilon) G_{f \sigma}(\omega+\varepsilon),
\end{aligned}
$$

with the auxiliary particle Green's functions, $G_{f \sigma}(\omega)=1 /\left[\omega+\mu-\lambda-E_{d}-\Sigma_{f \sigma}(\omega)\right]$ and $G_{b}(\omega)=1 /\left[\omega-\lambda-\Sigma_{b}(\omega)\right]$, respectively. $\lambda$ is a positive parameter, taken to $\lambda \rightarrow \infty$ in order to effect the constraint on the auxiliary particle number operator, $\sum_{\sigma} f_{\sigma}^{\dagger} f_{\sigma}+b^{\dagger} b=1$. Note that these NCA equations are coupled to the equations (6)-(9) for the interacting conduction electrons via the common chemical potential $\mu$ and via the conduction electron DOS of the interacting system in presence of a dilute, but finite impurity concentration, $A_{c \sigma}(\varepsilon)$. The Gd impurity electron Green's function is obtained from $G_{f \sigma}, G_{b}$ as,

$G_{d \sigma}(\omega)=\int \frac{d \varepsilon}{e^{\beta \varepsilon}}\left[A_{b}(\varepsilon) G_{f \sigma}(\varepsilon+\omega)-A_{f \sigma}(\varepsilon) G_{b}^{*}(\varepsilon-\omega)\right]$.

For an efficient and accurate method for numerically solving the set of equations (B1)-(B3) see Ref. [42].
* Email: kroha@physik.uni-bonn.de

1 G. Petrich, S. von Molnar, and T. Penney, Phys. Rev. Lett. 26, 885 (1971).

2 Y. Shapira, S. Foner, and T. B. Reed, Phys. Rev. B 8, 2299 (1973).

3 Y. Shapira, S. Foner, R. L. Aggarwal, and T. B. Reed, Phys. Rev. B 8, 2316 (1973).

${ }^{4}$ K. Y. Ahn and J. C. Suits, IEEE Trans Magnetics 3, 453 (1967).

5 J. Schoenes and P. Wachter, Phys. Rev. B 9, 3097 (1974).

${ }^{6}$ M. Matsubara, A. Schmehl, J. Mannhart, D. G. Schlom, and M. Fiebig, Phys. Rev. B 81, 214447 (2010).

7 M. Matsubara, A. Schmehl, J. Mannhart, A. Melville, D. G. Schlom, M. T. Martinez, A. Schroer, J. Kroha, and M. Fiebig, Nature Commun. 6, 6724 (2015).

8 P. G. Steeneken, L. H. Tjeng, I. Elfimov, G. A. Sawatzky, G. Ghiringhelli, N. B. Brookes, and D.-J. Huang, Phys. Rev. Lett. 88, 047201 (2002).

9 A. Schmehl, V. Vaithyanathan, A. Herrnberger, S. Thiel, C. Richter, M. Liberati, T. Heeg, M. Röckerath, L. F. Kourkoutis, S. Mühlbauer, et al., Nat Mater. 6, 882 (2007).

10 D. F. Förster, J. Klinkhammer, C. Busse, S. G. Altendorf, T. Michely, Z. Hu, Y.-Y. Chin, L. H. Tjeng, J. Coraux, and D. Bourgault, Phys. Rev. B 83, 045424 (2011).

11 J. Klinkhammer, D. F. Förster, S. Schumacher, H. P. Oepen, T. Michely, and C. Busse, Appl. Phys. Lett. 103, 131601 (2013).

12 A. G. Swartz, J. Ciraldo, J. J. I. Wong, Y. Li, W. Han, T. Lin, S. Mack, J. Shi, D. D. Awschalom, and R. K. Kawakami, Appl phys Lett. 97, 112509 (2010).

13 T. Kasuya, CRC Crit. Rev. Solid State Sci. 3, 131 (1972).

14 M. R. Oliver, J. O. Dimmock, A. L. McWhorter, and T. B. Reed, Phys. Rev. B 5, 1078 (1972).

15 T. Penney, M. W. Shafer, and J. B. Torrance, Phys. Rev. B 5, 3669 (1972).

16 A. Mauger, Phys. Stat. Solidi B 84, 761 (1977).

17 A. Mauger and C. Godart, Phys. Rep. 141, 51 (1986).

18 T. Matsumoto, , K. Yamaguchi, M. Yuri, K. Kawaguchi, N. Koshizaki, and K. Yamada, J. Phys. Condens. Matter 16, 6017 (2004).

19 H. Ott, S. J. Heise, R. Sutarto, Z. Hu, C. F. Chang, H. H. Hsieh, H.-J. Lin, C. T. Chen, and L. H. Tjeng, Phys. Rev. B 73, 094407 (2006).

20 T. Mairoser, A. Schmehl, A. Melville, T. Heeg, Canella, P. Böni, W. Zander, J. Schubert, D. E. Shai, E. J.
Monkman, et al., Phys. Rev. Lett. 105, 257206 (2010).

21 S. G. Altendorf, N. Hollmann, R. Sutarto, C. Caspers, R. C. Wicks, Y.-Y. Chin, Z. Hu, H. Kierspel, I. S. Elfimov, H. H. Hsieh, et al., Phys. Rev. B 85, 081201 (2012).

22 T. Mairoser, F. Loder, A. Melville, D. G. Schlom, and A. Schmehl, Phys. Rev. B 87, 014416 (2013).

23 S. G. Altendorf, A. Efimenko, V. Oliana, H. Kierspel, A. D. Rata, and L. H. Tjeng, Phys. Rev. B 84, 155442 (2011).

${ }^{24}$ M. Barbagallo, T. Stollenwerk, J. Kroha, N.-J. Steinke, N. D. M. Hine, J. F. K. Cooper, C. H. W. Barnes, A. Ionescu, P. M. D. S. Monteiro, J.-Y. Kim, et al., Phys. Rev. B 84, 075219 (2011).

25 M. Barbagallo, N. D. M. Hine, J. F. K. Cooper, N.-J. Steinke, A. Ionescu, C. H. W. Barnes, C. J. Kinane, R. M. Dalgliesh, T. R. Charlton, and S. Langridge, Phys. Rev. B 81, 235216 (2010).

26 S. Burg, V. Stukalov, and E. Kogan, Phys. Status Solidi B 249, 847 (2011).

27 N. Jutong, U. Eckern, T. Mairoser, and S. U., Scientific Reports 5, 8038 (2015).

28 M. A. Ruderman and C. Kittel, Phys. Rev. 96, 99 (1954).

29 T. Kasuya, Prog. Theor. Phys. 16, 45 (1956).

30 K. Yosida, Phys. Rev. 106, 893 (1957).

31 P. Sinjukow and W. Nolting, Phys. Rev. B 69, 214432 (2004).

32 M. Takahashi, Phys. Rev. B 86, 165208 (2012).

33 J. M. An and K. D. Belashchenko, Phys. Rev. B 88, 054421 (2013).

34 M. Arnold and J. Kroha, PRL 100, 046404 (2008).

35 N. Grewe and H. Keiter, Phys. Rev. B 24, 4420 (1981).

${ }^{36}$ Y. Kuramoto, Z. Phys. B 53, 37 (1983).

37 P. Coleman, Phys. Rev. B 29, 3035 (1984).

38 J. Kroha, Physica A 167, 231 (1990).

39 F. D. M. Haldane, Phys. Rev. Lett. 40, 416 (1978).

40 P. M. S. Monteiro, P. J. Baker, N. D. M. Hine, N.-J. Steinke, A. Ionescu, J. F. K. Cooper, C. H. W. Barnes, C. J. Kinane, Z. Salman, A. R. Wildes, et al., Phys. Rev. B 92, 045202 (2015).

41 Note, the reduction of the magnetic coupling observed in Ref. 7] only at high doping is indeed due to RKKY interaction, since the photodoping occurs not at the Fermi energy but into highly excited, short wavelength band states.

42 T. A. Costi, J. Kroha, and P. Wölfle, Phys. Rev. B 53, 1850 (1996). 